\title{
Evaluation of Selected Tree and Mesh based Routing Protocols
}

\author{
Harikrishna $T^{l}$, Dr.N.Geethanjali .A ${ }^{2}$ \\ ${ }^{1}$ Research Scholar in Rayalaseema University, Kurnool, \\ ${ }^{2}$ Professor department of computer science\& technology,SKU,Ananthapuramu.
}

\begin{abstract}
This paper research various routing protocols, problems and necessities comparatively in MANET routing and layout concerns which include classifications primarily based on layers and others. The layout and implementation of PUMA is a declarative constraint-fixing platform for coverage-based totally routing and channel selection in multiradio wi-fi mesh networks. PUMA integrates a high-performance constraint solver with a declarative networking engine. PUMA achieves a high data delivery ratio with very restricted manage overhead, which is nearly constant for a huge range of community situations. PUMA uses unattached manipulate packet format for querying the receivers while ODMR has separate manage packets for querying exclusive manipulate information. The outcomes from a huge range of eventualities of varying mobility, organization members, wide variety of senders, traffic load, and wide variety of multicast organizations display that PUMA attains higher packet delivery ratios than ODMRP and MAODV, whilst incurring some distance less manipulate overhead.
\end{abstract}

Keywords: PUMA Protocol, Multicast Routing, Control Packet, Manet Routing.

\section{INTRODUCTION}

Construction of pleasant viable multicast timber and retaining the organization connections in sequence is challenging even in stressed out networks. Though in MANETs multicast is one of the hard surroundings, the implementations of the tree based routing techniques are less complicated to the mesh based totally routing strategies. In tree based routing simplest unattached path is connecting the source node and free moving spot node, while in mesh primarily based routing, a couple of routes are connecting the supply node and vacation spot node. Tree-primarily based routing protocols be afflicted by the inferior overall performance of the mobility. If a link wreck happens then the statistics messages are misplaced until a brand new dimension is constructed. Multicast tree structures are without difficulty damaged and have to be readjusted continuously as connectivity changes. Furthermore, usual multicast trees regularly require global routing substructure. Frequent modifications of routing vectors or link nation table's reasons continuous changes in topology. As a result, it generates excessive channel and processing overhead. Limited bandwidth, limited strength, and mobility of community hosts make the design of multicast tree based totally routing protocols particularly hard. For this purpose it is a main trouble for the researchers to increase tree primarily based routing protocols. To remedy the essential problems inherent in tree-based tactics a new topology referred to as mesh has been proposed. The mesh topology is characterized via the fact that it affords multiple paths among a source and a receiver which lets in multicast records grams to be brought even though a hyperlink fails. A schematic way of layout and experimentation of NS2 configuring for crucial analysis. Here we taken into consideration protocols MAODV and ADMR of the tree based class and every other two protocols PUMA and ODMR of the mesh based elegance for illustrating protocol rating/ordering technique. However this contribution has a technological value and no longer having a lot throught nature. Above referred to protocols performances are considered for the QoS parameters which might be crucial for evaluating the worthiness of novel routing protocols which might be developed in this work.

\section{SELECTED TREE BASED ROUTING PROTOCOLS.}

A) Multicast Ad-hoc On-Demand Distance Vector Routing (MAODV) Protocol

MAODV is an improved model of AODV. It is dynamic, self-beginning, multi-hop routing protocol. MAODV creates a shared tree that is connecting the more than one sources and receivers in the multicast institution. The root of the each institution tree is both one of the multicast supply or receiver of the institution that has been designed as a Group Leader. The root is the primary member of a multicast group. When an utility on a node troubles a be a part of request for a multicast organization, this node floods the RREQ packet within the complete network. If no reaction is obtained from the group then the RREQ packet is repeated and the requested node will become the Group Leader for that institution. When a brand new supply wants to send packets to a collection, it plays the identical steps. This Group Leader takes the work of keeping the multicast group sequence variety. MAODV uses the unique collection range to recognize the multicast organization. Multicast Group Leader initializes the sequence variety and incremented at normal periods through a timer. By the use of modern-day collection number it generates the routes for multicast businesses.

After this the Group Leader floods the network through a Group Hello Packet to inform the community approximately the lifestyles of this Group and its modern series quantity. By the usage of the Group Hello Packet, the individuals within the institution update their request desk and distance to Group Leader. The MAODV discovers multicast routes On-Demand by using the use of broadcast discovery mechanisms i.E. Route Request and Route Reply. If nodes are asked to enroll in the institution or nodes want to ship packets to multicast organization then those nodes are required to get hold of Group Hello Packet from its Group Leader and unicast a RREQ packet to the Group Leader.

Once the Group Leader gets the RREQ packet it uncast a 
RREP packet again to the originator of the RREQ which responds with a multicast activation (MACT) packet. The MACT packet establishes multicast forwarding country between the newly joined receiver and the shared tree. If a source node does not receive a MACT within a sure time frame then it broadcast any other RREQ. After the quantity of RREQs, the supply assumes that there aren't any different members of the tree that can be reached and announces itself as the Group Leader. In this damaged links are detected with the assist of periodic Hello packets transmitted through every node inside the community and nodes use the increasing ring search mechanism to reconnect the shared tree.

\section{B) Adaptive Demand Driven Multicast Routing (ADMR) Protocol}

ADMR is a receiver initiated multicast tree. If at the least one supply and one receiver are lively for the organization it creates a tree via using an on-demand mechanism. ADMR helps receivers to receive multicast packets dispatched through any sender. As well as receivers might also be part of a multicast institution dealt on behalf of unique senders. The multicast source does now not understand who are the receivers and in which community they may be located. The receivers want now not realize who are the assets and wherein network they are placed. ADMR works with the nodes which might flow at any time within the complete community and any packet is lost inside of network. To be part of a multicast organization, an ADMR receiver transmits a MULTICAST SOLICITATION packet in the entire community. Once source receives this packet, it replies through sending a unicast KEEP-ALIVE packet to that receiver and additionally to confirm that the receiver has joined that source. The receiver replies to the KEEP-ALIVE through sending a RECEIVER JOIN packet alongside the reverse direction which units up forwarding state along the shortest paths. Additionally to the receiver's join mechanism, a source floods the RECEIVER DISCOVERY packet periodically inside the entire community. The Receivers which exist in the community and if there is no longer already related to the multicast tree then they get this packet and reply to it with a RECEIVER JOIN packet. Within the tree to locate broken hyperlinks, supply monitors the packet forwarding charge to determine while the tree has damaged or the supply node has emerge as silent. When the hyperlink break occurs then the node initiates a repair on its personal. If the source node stops sending the packets then any forwarding country is silently eliminated. Receivers equally screen the packet reception rate and understand the way to re-join the multicast tree if intermediate nodes had been not able to reconnect the tree. The receivers ensure a restore by means of broadcasting a new MULTICAST SOLICITATION packet. On the opposite hand a node at the multicast tree transmits a REPAIR NOTIFICATION packet down its sub-tree to cancel the restore of downstream nodes. The most upstream node sends a hop-constrained flood of a RECONNECT packet. If any forwarder receives this packet then it forwards the RECONNECT up the multicast tree to the source. The supply responds to the RECONNECT packet through sending a RECONNECT REPLY as a unicast message that follows the route of the RECONNECT returned to the repairing node. Thus, it performs each its direction discovery and route renovation features on demand.

\section{SELECTED MESH BASED ROUTING PROTOCOLS.}

\section{A) On-Demand Multicast Routing (ODMR) Protocol}

It is a mesh-based totally multicast routing protocol. To create a mesh for each multicast organization ODMR protocol $[12,51]$ makes use of the method of forwarding group [43]. This protocol establishes multicast routes and organization on-demand and it is brought to the source. ODMR protocol makes use of course request and reply phase. In the network if a source node has packets to ahead then it periodically declares a member advertising and marketing packet called a Join Query. If a node gets a Join Query then it collects the upstream node ID and additionally rebroadcasts the packet. If the Join Query packet reaches multicast receiver then the receiver creates or updates the supply entry in its Member Table. When legitimate entries present inside the Member Table then Join Replies are broadcasted periodically to its nearest node. While a node receives Join Query it tests if the next node ID is one of the entries matches together with its own ID. If the node realizes that it is in the direction to the supply and a phase of the forwarding institution then it broadcast its very own Join Reply. Every forwarding group member sends the Join Reply until it reaches the multicast source via the shortest course. In the forwarding institution this manner may be used to create or replace the routes taking off from sources to receivers and paperwork a mesh of nodes. After the formation of the institution alongside the direction production procedure a multicast supply sends packets to receivers via desired routes and forwarding businesses. Periodic control packets are brought handiest whilst outgoing records packets are nevertheless there. While receiving a multicast information packet a node sends packets simplest while it isn't always a replica. To join or depart the institution no specific manipulate packets require to be sent. In case a multicast supply desires to leave the institution it stops sending Join Query packets immediately because it is not having any multicast statistics to ahead to the institution. From a particular multicast group a receiver which no longer wants to receive, the receiver gets rid of the subsequent entries from its Member Table and want not transmit the Join Reply for that organization.

\section{B) Protocol for Unified Multicasting thru Announcements} (PUMA)

PUMA is a mesh based routing protocol which supports a source node to transmit multicast packets addressed to a known multicast group without having the information of the way the group is. Within the community it selects one of the receivers of a collection as middle of the organization .In addition it informs every router as at the least one subsequent-hop closer to the selected center of each organization. Every node on shortest route connecting any receiver and the core, shape a mesh. Sender transmits a facts packet to the group with any of the shortest paths connecting the sender and the core. Once the statistics packet reaches a mesh member it floods within the mesh. In addition, nodes maintain a packet ID cache to drop duplicate packets.

PUMA makes use of single control packet for each function i.E. Multicast Announcement Packet (MAP). Every MAP has a series variety, group ID (address of the organization), core ID (deal with of the center), distance to the middle, and 
mesh member flag and determine to choose a neighbor to reach the middle. Succeeding MAPs' contain better collection number than in advance multicast announcements dispatched by way of the equal center. By manner of this information nodes pick cores and locate the routes for sources past a multicast and additionally provide the information concerning joining or leaving the mesh of a set and preservation of the mesh. A node in the organization that's core of a collection periodically transmits the multicast announcement. When the multicast statement travels for the duration of the community, it creates a connectivity listing at each node inside the community. By method of connectivity lists nodes want to create a mesh and also direction the statistics packets from senders to receivers. A node collects the facts from each multicast announcement and it accepts from its nodes in the connectivity list. A new multicast statement from a neighbor that's having higher collection wide variety overwrites with that of a lower sequence wide variety for the identical institution. For a given institution, a node has only one access in its connectivity list from a specific neighbor and it keeps most effective those facts with the modern day sequence range for a given core.

Every access in the connectivity listing collects the records concerning the multicast declaration, the time when it become acquired and the neighbor from which it changed into received. The node creates its personal multicast declaration primarily based on the excellent entry within the connectivity listing. For the similar center ID and maximum sequence variety the multicast announcements via smaller distances to the center are considered pleasant. Connectivity list and document work a recent list that's constrained to the new core If each and each subject is equal then the multicast declaration that arrived formerly is taken into consideration. After figuring out the satisfactory multicast declaration packet, the node creates its own multicast declaration packet. The connectivity list collects records concerning all the routes that exist to the center. If a core change takes place for a particular institution then the node removes the entries of its old.

\section{PERFORMANCE EVALUATION THROUGH SIMULATION}

In order to evaluate the performance of a routing protocol we want to apply a community simulator like NS-2, Qual Net, OPTNET and GloMoSim and so on. In this, we used NS-2 to create the simulation environment on the way to implement the protocols and additionally to compare its overall performance with each other

\section{a) Performance metrics}

For finding the overall performance Evaluation of the selected protocols we recollect throughput, packet shipping ratio and postpone Throughput is defined as the whole common variety of facts packets acquired by way of the destination in bytes per second. Packet Delivery ratio is the ratio of the data packets obtained at the destination to the variety of records packets transmitted by means of the supply. Average End-to-End Delay represents the common time i.E. The transmission put off of facts packets which might be introduced correctly. This postpone consists of propagation delays, queuing delays on the interfaces and buffering delays at some point of path discovery.

\section{B) Simulation Scenario \& Scenario Setup}

The simulator for evaluation of proposed routing protocol is carried out the usage of Network Simulation (NS2) in Linux. The network size is of 50-200 nodes incrementing via 50 nodes placed randomly over $1100 \mathrm{~m} \times 1100 \mathrm{~m}$ vicinity. The transmission variety for each node is $250 \mathrm{~m}$, and the channel potential is $2 \mathrm{Mbits} / \mathrm{s}$. The mobility version of the nodes within the simulations is the Random Waypoint version. Every node alone begins at a random position in the simulation region and stays desk bound for an interval of pause time. The node then generates a uniformly dispensed latest location, which is a random destination within the simulation place. The mobility speed is uniformly dispensed between precise mobility speeds of $0 \mathrm{mts} / \mathrm{sec}$ to $10 \mathrm{mts} / \mathrm{sec}$, with a pause time of 0 seconds equivalent to a regular motion of $10 \mathrm{sec}$.

\section{C) Channel and Radio Model}

The propagation models in NS2 have loose area version, -ray floor mirrored image model and the shadowing version [59]. Free area model assumes the precise scenario in which handiest one clean line-of-sight route some of the transmitter and receiver. The two were ground reflection model considers the direct path and floor mirrored image course together. The shadowing version consists of parts i.E. The first component is direction loss example, mobile nodes may not move in straight lines at regular speeds for the whole simulation due to the fact actual cell nodes could no longer pass in such a confined way. There are unique sorts of mobility fashions which might be to be had i.E. Random mobility model, Group mobility version, Temporal mobility model and Spatial mobility model Again in the Random fashions, Random stroll mobility version, Random Waypoint mobility model, Random Direction mobility etc. Are available. In the proposed protocols we consider the broadly used Random Point route mobility model [for the overall performance of the protocols.

\section{D) Random Waypoint Mobility Model}

Random route point Mobility Model comprises the pause times which entails the modifications in the direction and/or velocity. A node starts offevolved in staying at one function intended for a particular time frame i.E. Pause time. When this time expires the cellular node selects a random destination within the simulation vicinity and the velocity that's uniformly distributed the various most pace and minimal speed. The cellular node moves inside the direction of the newly selected destination with the aid of the chosen pace. On arrival, the cellular node stops for a selected time period previous to beginning the system again.

In most of the performance evaluation that use the Random Waypoint Mobility Model, the mobile nodes are at first disbursed randomly at some stage in the simulation vicinity. The random distribution of model and the second one element reflects the version of the received strength at unique distance. The two-ray ground reflection model is used for simulation inside the proposed protocol.

\section{E) MAC Protocol}

The IEEE 802.Eleven MAC protocol thru allotted coordination feature (DCF) is used as the MAC layer. DCF uses a RTS / CTS / DATA /ACK for all unicast packets. For multicast data packets they're sending without using ACK within the series.

F) Simulation Parameters and Traffic scenario 
The selected protocols are evaluated the use of Network simulator (NS2) of fifty-2 hundred nodes incrementing through 50 nodes. The mobility version is chosen as Random Way Point model. In this mobility model a node randomly selects a destination and it moves in the direction of the vacation spot with a velocity uniformly chosen among the minimum pace and maximal velocity. After it reaches the destination, the node remains there for a pause time after which actions again. Each node moves randomly with a pace of zero- $10 \mathrm{mts} / \mathrm{sec}$ and stays at the identical region with a pause time zero-10sec. The Distributed Coordinated Function (DCF) of IEEE 802.11 for wi-fi LANs is believed as the MAC layer protocol. The two ray ground version is selected for the propagation. A bandwidth of $2 \mathrm{Mbps}$ with a radio variety of $250 \mathrm{~m}$ is taken into consideration. We have elected CBR as the form of verbal exchange and the maximum interface queue period is 250. The overall performance metrics considered are Throughput, Average End-to-End Delay and Packet Delivery Ratio.

\section{SIMULATION RESULTS}

We evaluated and as compared the overall performance of the tree based totally routing protocols i.E. ADMR and MAODV routing protocol, mesh based totally routing protocols i.E. ODMR and PUMA. We have taken into consideration the various node mobility, various node density and their group sizes. In this we decided on a network with node mobility starting from $2 \mathrm{mts} / \mathrm{sec}$ to $10 \mathrm{mts} / \mathrm{sec}$ incrementing the steps of two, node density degrees from 50-2 hundred which increments within the steps of fifty and their institution sizes in with increments of 1. Thus the feasible mixtures for comparing above 4 protocols is 8 i.E. No. Of distinct densities taken into consideration extended with out a. Of distinct businesses considered (2). The consequences for different overall performance metrics plotted inside the graph for different parameters and node mobility are illustrated in Fig 3.1 to 3.Three. In the overall performance analysis throughput, packet shipping ratio and end-to-end delay comparisons are offered inside the subsequent 3 sub sections.

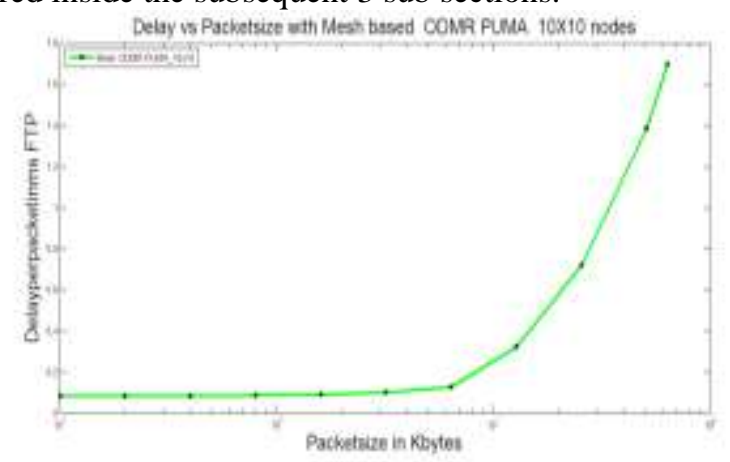

Fig 1.Delay vs throughput dimension

\section{a. Throughput}

By gazing the graphs plotted in Fig.1, you may be aware that once the wide variety of agencies equal to at least one, PUMA is slightly higher than ODMR and in turn ODMR is better than MAODV and ADMR. As the organization depend is elevated from one to two, PUMA is a good deal higher than ODMR. This behavior is because of the distinction within the format of the manipulate packets used.
PUMA uses single manipulate packet format for querying the receivers while ODMR has separate manipulate packets for querying specific control information. Thus the manage packets exchanged among sender and receivers are greater compared to records packets in ODMR than PUMA. Hence the result of throughputs is fewer in ODMR. PUMA offers higher throughputs because it focuses on mesh redundancy in the region.

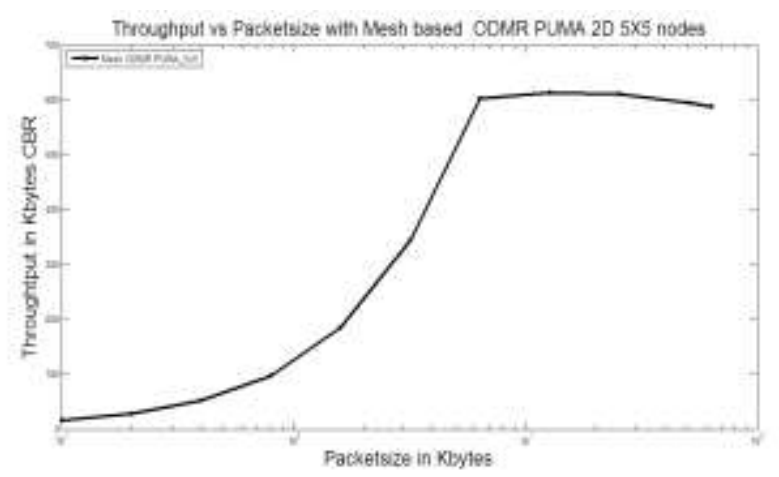

Fig2: Throughput Vs Packet size

\section{b.Packet Delivery Ratio}

By gazing the graphs plotted in Fig.2, you could word the risky transport ratios exhibited by using ADMR for the diverse densities and institution counts considered except the density equals to 200. But its conduct is terrible compared to all different three protocols because of the reality that more number of link disasters came about in ADMR. The ODMR presentations good following ratios with organization be counted equals to at least one in comparison to MAODV. But with the number of companies equal to 2, the transport ratios of ODMR and MAODV are nearly identical and slightly ruled by way of ODMR.

\section{c.End to End Delay}

Fig. 3 conveys the reality that the give up-to-stop delay of ADMR is higher in comparison to MAODV except few instances wherein both of them are overlapping with every different. This is because of the facts that during ADMR the receiver needs to send a affirmation to every supply. The give up-to-cease delays in PUMA are lesser in comparison to the other 3 protocols and ODMR is dominating MAODV and ADMR. But with grouping depend equals to two, ODMR shows higher delays as compared to all different three protocols taken into consideration for evaluation. But PUMA nevertheless presentations regular quit-to-give up delays.

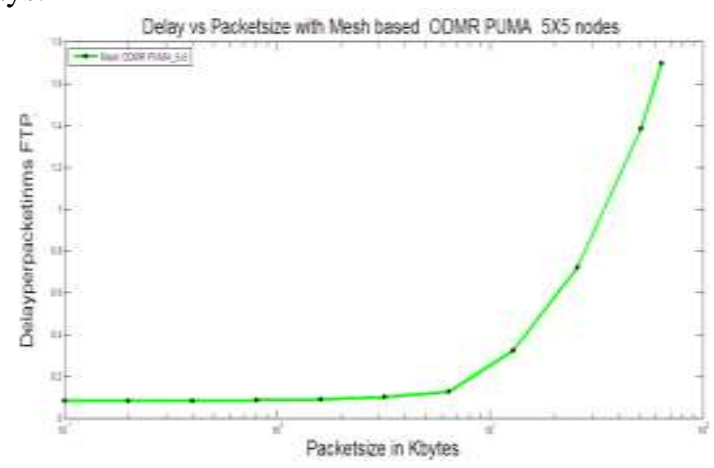

Fig3: Deliver Ratio for PUMA 


\section{REFERENCES}

[1] D.P Agarwal and Qing-An Zeng. "Introduction to wireless and Mobile Systems", Brooks/Cole, 2005.

[2] R.G.T.Anderson, B.Bershad and D.Wetherall. "A System Architecture for pervasive computing", In Proc. 9th ACM SIGOPS European Workshop, pp.177-182, Kolding, Denmark, September 2000.

[3] I. Chlamtac and J. Redi. "Mobile Computing: Challenges and Apportunities", A.Ralston and E.Reilly (Eds), International Thomson Publishing, 1998.

[4] A. K. Verma, Mayank Dave and R. C. Joshi. "Secure Routing in Mobile Networks: A Review", International Journal of Systematics, Cybernatics and Informatics (IJCSI), ISSN 0973-4864, 2007.

[5] A. Perrig, R. Canetti, J. D. Tygar and D. Song. "Efficient Authentication and Signing of Multicast Streams over Lossy Channels", In Proc. of IEEE Symposium on Security and Privacy, 2000.

[6] S. Corson and J. Macker. "Mobile ad hoc networking (MANET): Routing protocol performance issues and evaluation considerations", RFC 2501, January 1999, available at ttp://www.ietf.org/rfc/rfc2501.txt.

[7] Zhigang WANG, Lichuan LIU and Meng Chu ZHOU. "Protocols and Applications of Ad-hoc Robot Wireless Communication Networks: An Overview", International Journal of Intelligent Control and Systems, vol. 10, no. 4, December 2005, 296-3 8.Architectures and Protocols", Prentice-Hall, Upper Saddle River, NJ, USA, 2004.

[8] Charles E. Perkins. "Ad hoc Networking", Pearson Education Inc., 2008.

[9] E. M. Royer and Charles E. Perkins. "Multicast operation of the ad-hoc on-demand distance vector routing protocol", Proceedings Of the 5th ACM/IEEE International Conference on Mobile Computing and Networking, Mobicom'99, pp. $207-$ 218, August 1999.

[10] J. G. Jetcheva and D. B. Johnson. "Adaptive demanddriven multicast routing in multi-hop wireless ad hoc networks", in Proceedings of the 2nd ACM International Symposium on Mobile Ad Hoc Networking and Computing (MobiHoc '01), pp. 33-44, 2001.

[11] R. Vaishampayan and J. J. Garcia-Luna-Aceves. "Protocol for Unified zMulticasting through Announcements (PUMA)", in Proceedings of the IEEE International Conference on Mobile Ad-Hoc and Sensor Systems (MASS '04), 2004.

[12] M. Nagaratna, C. Raghavendra Rao, V. Kamakshi Prasad. "Node Disjoint Split Multipath Protocol for Unified Multicasting through Announcements (NDSM-PUMA)", Proceedings of International Conference on Computer Science and Information Technology CSIT-2011, pp 373-380, Bangalore, India, July 2011.

[13] B.Dahill, B.Levine, E.Belding Royer and C. Shields. "A Secure Routing protocol for Ad hoc Networks", University of Massachusetts Technical Report, pp 01-37, 2001.

[14] C.-K. Toh. "Ad Hoc Mobile Wireless Networks: Protocols and Systems", Prentice-Hall, Englewood Cliffs, NJ, USA, 2002.

[15] S. Paul. "Multicasting on the Internet and Its Applications", Kluwer Academic Publishers, Norwell, Mass, USA, 1998. [28]. I. Stojmenovi'c Ed., "Handbook of Wireless Networks and Mo bile Computing, "John Wiley \& Sons, New York, NY, USA, 2002.1989.

[16] Osamah S. Badarneh and Michel Kadoch. "Multicast Routing Protocols in Mobile Ad Hoc Networks: A Comparative Survey and Taxonomy", Hindawi Publishing Corporation EURASIP Journal on Wireless Communications and Networking Volume 2009, Article ID 764047, 42 pages doi:10.1155/2009/764047.

[17] L. Junhai, X. Liu, and Y. Danxia. "Research on multicast routing protocols for mobile ad-hoc networks", Computer Networks, vol. 52, no. 5, pp. 988-997, 2008.

[18] C. C. Chiang, M. Gerla, and L. Zhang. "Adaptive shared tree multicast in mobile wireless networks", in Proceedings of the IEEE Global Telecommunications Conference (GLOBECOM'98), vol. 3, pp. 1817-1822, 1998.

[19] C. C. Shen and C. Jaikaeo. "Ad hoc multicast routing algorithm with swarm intelligence", Mobile Networks and Applications, vol. 10, no. 1, pp. 47-59, 2005.

[20] S. Park and D. Park. "Adaptive core multicast routing protocol" Wireless Networks, vol. 10, no. 1, pp. 53-60, 2004.

[21] C. K. Toh, G. Guichal and S. Bunchua. "ABAM: ondemand associativity-based multicast routing for ad hoc mobile networks", in Proceedings of the IEEE Vehicular Technology Conference (VTC '00), vol. 3, pp. 987-993, 2000. [22] T. Ozaki, J. B. Kim and T. Suda. "Bandwidth-efficient multicast routing for multihop, ad-hoc wireless networks", in Proceedings of the 20th Annual Joint Conference of the IEEE Computer and Communications Societies (INFOCOM'01), vol. 2, pp. 1182-1191, 2001.

[23] C. C. Hu, E. H. K. Wu, and G. H. Chen. "OGHAM: on demand global hosts for mobile ad-hoc multicast services", Ad Hoc Networks, vol. 4, no. 6, pp. 709-723, 2006.

[24] D.Pompili and M. Vittucci. "PPMA, a probabilistic predictive multicast algorithm for ad hoc networks", Ad Hoc Networks, vol. 4, no. 6, pp. 724-748, 2006.

[25] J. Xie, R. R. Talpade, A. McAuley, and M. Liu. "AMRoute: ad hoc multicast routing protocol", Mobile Networks and Applications, vol. 7, 132 no. 6, pp. 429-439, 2002.

[26] M. Ge, S. V. Krishnamurthy, and M. Faloutsos. "Application versus network layer multicasting in ad hoc networks: the ALMA routing protocol", Ad Hoc Networks, vol. 4, no. 2, pp. 283-300, 2006.

[27] Ye Z, Krishnamurthy SV and Tripathi SK. "A frame work for reliable routing in mobile ad hoc networks", in Proceedings of the 22nd annual joint conference of the IEEE computer and communications societies (INFOCOM), vol.1,2003, p.270-80.

[28] Mueller S and Ghosal D. "Analysis of a distributed algorithm to determine multiple routes with path diversity in ad hoc networks", Proceedings of the third international symposium on modeling and optimization in mobile, ad hoc and wireless networks(WIOPT), 2005, p.277-85.

[29] An H-Y, Zhong L, LuX-C, Peng W. "A cluster-based multipath dynamic source routing in MANET", Proceedings of the IEEE international conference on wireless and mobile computing, networking and communications (WIMOB), 2005, vol.3, p.369-76.

[30] Wisitpongphan N, Tonguz OK. "Disjoint multipath source routing in ad hoc networks: transport capacity", Proceedings of the IEEE 58th vehicular technology conference (VTC), vol.4, October2003, p.2207-11.

[31] R.Bagrodia, M. Gerla, J. Hsu, W. Su, and S. J. Lee. “A performance comparison study of ad hoc wireless multicast protocols", Proc. of the 19th ComSoc IEEE Conference, pp. $565-574$, (2000). 